\title{
Scaling behavior of the order parameter and its conjugated field in an absorbing phase transition around the upper critical dimension
}

\author{
S. Lübeck网 \\ Theoretische Tieftemperaturphysik, Gerhard-Mercator-Universität, Lotharstr. 1, 47048 Duisburg, Germany
}

(Dated: Dezember 12, 2001)

\begin{abstract}
We analyse numerically the critical behavior of an absorbing phase transition in a conserved lattice gas in an external field. The external field is realized as a spontaneous creation of active particles which drives the system away from criticality. Nevertheless, the order parameter obeys certain scaling laws for sufficiently small external fields. These scaling laws are investigated and the corresponding exponents are determined in various dimensions $(D=2,3,4,5)$. At the so-called upper critical dimension $D_{\mathrm{c}}=4$ one has to modify the usual scaling laws by logarithmic corrections.

PACS numbers: 05.70.Ln, 05.50.+q, 05.65.+b
\end{abstract}

\section{INTRODUCTION}

Recently Rossi et at. introduced a conserved lattice gas (CLG) with a stochastic short range interaction that exhibits a continuous phase transition from an active state to an absorbing non-active state at a critical value of the particle density [1]. The CLG model is expected to belong to a new universality class of absorbing phase transitions characterized by a conserved field. Similar to the well known universality hypothesis of directed percolation [2, 3] the authors conjectured that "all stochastic models with an infinite number of absorbing states in which the order parameter evolution is coupled to a nondiffusive conserved field define a unique universality class" [1]. In order to check this hypothesis the scaling behavior, i.e. the values of the corresponding critical exponents, has to be determined. The order parameter exponent as well as the exponent of the order parameter fluctuations were determined in 何. Furthermore a modified CLG model with random particle hopping was introduced in [5] which mimics the mean-field scaling behavior of the system.

In this work we investigated for the first time the CLG model in an external field. The external field is conjugated to the order parameter, i.e. it is realized as a spontaneous creation of active particles. Of course a spontaneous creation of active particles destroys the absorbing state and thus the absorbing phase transition at all. But analogous to ferromagnetic phase transitions the order parameter can be described as a generalized homogeneous function of the control parameter and of the conjugated field in the critical regime. This scaling behavior is examined below $(D=2,3)$, above $(D=5)$, and at the so-called upper critical dimension $(D=4)$.

*Electronic address: sven@thp.uni.duisburg.de

\section{MODEL AND SCALING BEHAVIOR}

We consider the CLG model on $D$-dimensional cubic lattices of linear size $L$. Initially one distributes randomly $N=\rho L$ particles on the system where $\rho$ denotes the particle density. In order to mimic a repulsive interaction a given particle is considered as active if at least one of its $2 D$ neighboring sites on the cubic lattice is occupied by another particle. If all neighboring sites are empty the particle remains inactive. Active particles are moved in the next update step to one of their empty nearest neighbor sites, selected at random. In the steady state, the system is characterized by the density of active sites $\rho_{\mathrm{a}}$. The density $\rho_{\mathrm{a}}$ is the order parameter of the absorbing phase transition, i.e., it vanishes if the control parameter $\rho$ is lower than the critical value $\rho_{\mathrm{c}}$. In the thermodynamic limit the order parameter scales for zero field and for $\rho>\rho_{\mathrm{c}}$ as

$$
\rho_{\mathrm{a}}(\delta \rho, h=0) \sim \delta \rho^{\beta}
$$

with $\delta \rho=\rho / \rho_{\mathrm{c}}-1$.

In this work we consider the CLG model in an external field $h$ conjugated to the order parameter, i.e., the field spontaneously creates active particles. Clearly the particular implementation of the spontaneous creation of active particles has to obey the particle conservation of the CLG model which is believed to be a relevant parameter, determining the universality class. Instead of a creation of additional particles we realize the external field by particle movements which do not change the total number of particles. Therefore we choose randomly $L^{D} h$ particles on the lattice. Each of these particles is then moved to one of its empty next neighbors. In this way inactive particles may be activated and the number of active sites is increased.

In our simulations we start with randomly distributed particles. The system is updated according to the above rules in the following way. One lists all active sites and updates these sites in a randomly chosen sequence. Then one moves $L^{D} h$ randomly chosen inactive particles to one of its empty next neighbors. In the case that these movements create active particles, these particles are added to 


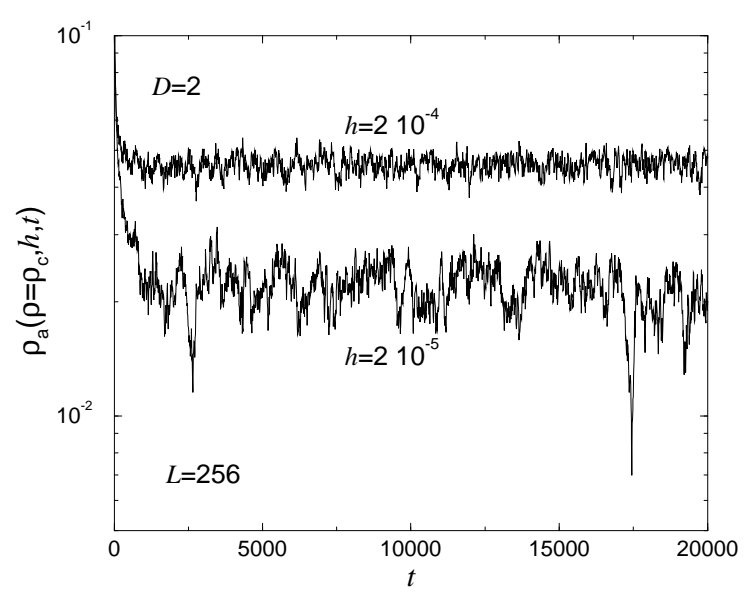

FIG. 1: The density of active sites $\rho_{\mathrm{a}}$ as a function of time (number of update steps) for two different values of the external field $h$. After a certain relaxation time the density of active sites fluctuates around a well defined value. For too small fields finite-size effects occur which results in strong fluctuation events (see lower curve).

the list of active particles and will be updated in the following update step. Thus one update step contains both, the update of active sites and the additional movements of inactive particles which mimics the external field.

In Fig. 1 we plot the density of active sites vs. time (number of update steps) at $\rho_{\mathrm{c}}$ for two different values of the driving field. After a certain relaxation time the system reaches a steady state where the density of active sites fluctuates around the average value $\left\langle\rho_{\mathrm{a}}(\delta \rho, h, t)\right\rangle$ which is interpreted as the order parameter $\rho_{\mathrm{a}}(\delta \rho, h)$. Decreasing the external field below a certain value finite-size effects occur which results in strong fluctuations. Two of these strong fluctuations events can be seen in the lower curve of Fig. 1. The origin of these effects is that the number of particle movements $\left(L^{D} h\right)$ create too few active particles and the system tends to the absorbing state. As a results the measured order parameter is shifted to lower values. To avoid these finite-size effects we increase the system size before these fluctuation events occur.

The spontaneous creation of active particles destroys the absorbing state and thereby the phase transition itself. Although the external field drives the system away from criticality the order parameter obeys certain scaling laws for sufficiently small fields. At the critical density the order parameter is expected to scale with the field as (see for instance [6])

$$
\rho_{\mathrm{a}}(\delta \rho=0, h) \sim h^{\beta / \sigma} .
$$

As usual in critical phenomena the order parameter is assumed to be a generalized homogeneous function of the control parameter $\delta \rho$ and the applied field $h$

$$
\rho_{\mathrm{a}}(\delta \rho, h)=\lambda \tilde{r}\left(\delta \rho \lambda^{-1 / \beta}, h \lambda^{-\sigma / \beta}\right)
$$

with the scaling function $\tilde{r}_{\text {. }}$ Choosing $\delta \rho \lambda^{-1 / \beta}=1$ at zero field one recovers Eq. (11) whereas $h \lambda^{-\sigma / \beta}=1$ leads to Eq. (2) at the critical density, respectively.

Furthermore we consider the fluctuations of the order parameter

$$
\Delta \rho_{\mathrm{a}}(\delta \rho, h)=L^{D}\left[\left\langle\rho_{\mathrm{a}}(\delta \rho, h, t)^{2}\right\rangle-\left\langle\rho_{\mathrm{a}}(\delta \rho, h, t)\right\rangle^{2}\right] .
$$

For zero field the fluctuations are known to diverge approaching the critical point [4]

$$
\Delta \rho_{\mathrm{a}}(\delta \rho, h=0) \sim \delta \rho^{-\gamma^{\prime}}
$$

The fluctuation exponent $\gamma^{\prime}$ fulfill the scaling relation [7]

$$
\gamma^{\prime}=\nu_{\perp} D-2 \beta
$$

where the exponent $\nu_{\perp}$ describes how the spatial correlation length diverges at the transition point. In the critical regime we assume that the fluctuations obey the scaling ansatz

$$
\Delta \rho_{\mathrm{a}}(\delta \rho, h)=\lambda^{\gamma^{\prime}} \tilde{d}\left(\delta \rho \lambda, h \lambda^{\sigma}\right)
$$

Setting $\delta \rho \lambda=1$ one recovers Eq. (5) for $h=0$.

Analogous to equilibrium phase transitions the susceptibility is defined as the derivative of the order parameter with respect to the conjugated field

$$
\begin{aligned}
\chi(\delta \rho, h) & =\frac{\partial}{\partial h} \rho_{\mathrm{a}}(\delta \rho, h) \\
& =\lambda^{1-\sigma / \beta} \tilde{c}\left(\delta \rho \lambda^{-1 / \beta}, h \lambda^{-\sigma / \beta}\right) .
\end{aligned}
$$

Approaching the transition point the susceptibility diverges for zero field as

$$
\chi(\delta \rho, h=0) \sim \delta \rho^{-\gamma} .
$$

This result can be recovered from Eq. (8) by setting $\delta \rho \lambda^{1 / \beta}=1$ for $h=0$ and one gets the scaling relation

$$
\gamma=\sigma-\beta
$$

which corresponds to the well known Widom equation of equilibrium phase transitions. Using this scaling relation we calculate in the following the value of the susceptibility exponent $\gamma$ from the obtained values of $\beta$ and $\sigma$. Notice that in contrast to the scaling behavior of equilibrium phase transitions the non-equilibrium absorbing phase transition is characterized by $\gamma \neq \gamma^{\prime}$.

\section{BELOW THE CRITICAL DIMENSION}

\section{A. $\mathrm{D}=2$}

At the beginning of our analysis we consider the twodimensional CLG model at the critical density $\rho_{\mathrm{c}}$ for various values of the driving field $h$ where the value of the critical density is obtained from [4]. The field dependence 


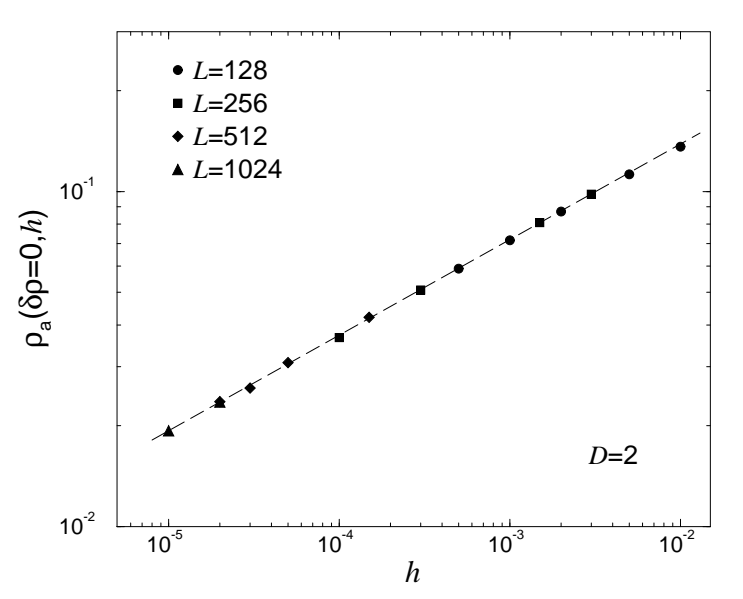

FIG. 2: The order parameter $\rho_{\mathrm{a}}$ as a function of the field $h$ at the critical density $\rho=\rho_{\mathrm{c}}$ for $D=2$. The dashed line corresponds to a power-law behavior according to Eq. (2) with an exponent $0.286 \pm 0.001$.

of the order parameter $\rho_{\mathrm{a}}(\delta \rho=0, h)$ is shown in a loglog plot Fig.2. Approaching the critical point $(h \rightarrow 0)$ the order parameter vanishes algebraically in agreement with Eq. (2). A regression analysis of the data yields the estimation $\beta / \sigma=0.286 \pm 0.001$. Using the value of the order parameter exponent $\beta=0.637 \pm 0.009$ [四] we get $\sigma=2.227 \pm 0.032$.

In the following we analyse the order parameter as a

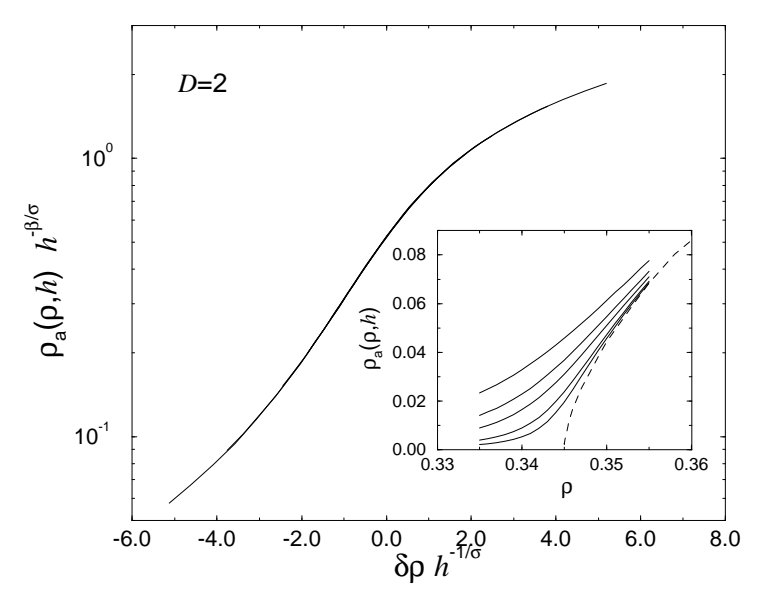

FIG. 3: The scaling plot of the order parameter $\rho_{\mathrm{a}}$ for the two-dimensional model. The data are rescaled according to Eq. (11) with $\beta=0.637$ and $\sigma=2.227$. The inset displays the unscaled data, i.e., the order parameter $\rho_{\mathrm{a}}$ is plotted as a function of the density $\rho$ for different values of the field $h$. The order parameter is driven away from the critical point $\left(\rho_{\mathrm{c}}, 0\right)$ with increasing field. The dashed line corresponds to the zero-field behavior.

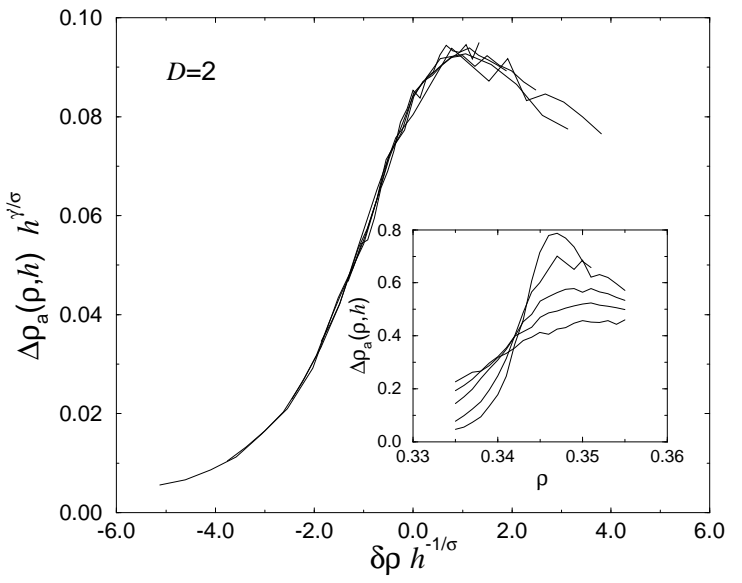

FIG. 4: The scaling plot of the order parameter fluctuations $\Delta \rho_{\mathrm{a}}$ for the two-dimensional model. The data are rescaled according to Eq. (12) with $\sigma=2.227$ and $\gamma^{\prime} / \sigma=$ 0.18 . The inset displays the unscaled data, i.e., the fluctuations are plotted as a function of the density $\rho$ for different values of the field $h$. Approaching the transition point $(h \rightarrow 0$ at $\rho=\rho_{\mathrm{c}}$ ) the peak of the fluctuations diverges.

function of the control parameter $\delta \rho$ for different fields from $h=10^{-5}$ up to $210^{-4}$. The applied field results in a rounding of the zero-field curve, i.e., the order parameter increases smoothly with the control parameter for $h>0$ (see inset of Fig. 33). According to the scaling ansatz of the order parameter [Eq. (3)] we choose $h \lambda^{-\sigma / \beta}=1$ and get the scaling form

$$
\rho_{\mathrm{a}}(\delta \rho, h)=h^{\beta / \sigma} \tilde{r}\left(\delta \rho h^{-1 / \sigma}, 1\right) .
$$

Thus plotting $\rho_{\mathrm{a}} h^{-\beta / \sigma}$ as a function of $\delta \rho h^{-1 / \sigma}$ the curves for different values of the driving field have to collapse onto the scaling function $\tilde{r}$. Using the above determined values of $\beta$ and $\sigma$ one gets an excellent data collapse which is shown in Fig. 3 .

Next we consider the scaling behavior of the order parameter fluctuations $\Delta \rho_{\mathrm{a}}$. The fluctuation data are shown for different values of the external field in the inset of Fig. A. For finite fields the fluctuations display a peak. Approaching the transition point $(h \rightarrow 0)$ this peak becomes a divergence signalling the critical point. In order to analyze the scaling behavior of the fluctuations we use Eq. (7) and set $h \lambda^{\sigma}=1$ which yields

$$
\Delta \rho_{\mathrm{a}}(\delta \rho, h)=h^{-\gamma^{\prime} / \sigma} \tilde{d}\left(\delta \rho h^{-1 / \sigma}, 1\right) .
$$

Since $\rho_{\mathrm{c}}$ and $\sigma$ are already determined one can estimate the exponent $\gamma^{\prime}$ by varying this exponent until one observes a data collapse of the different fluctuation curves. The best result is obtained for $\gamma^{\prime} / \sigma=0.18 \pm 0.02$, leading to $\gamma^{\prime}=0.402 \pm 0.045$, and the corresponding plot is shown in Fig. A. The value agrees with $\gamma^{\prime}=0.384 \pm 0.023$ [ [1] obtained from a regression analysis according to Eq. (5). 


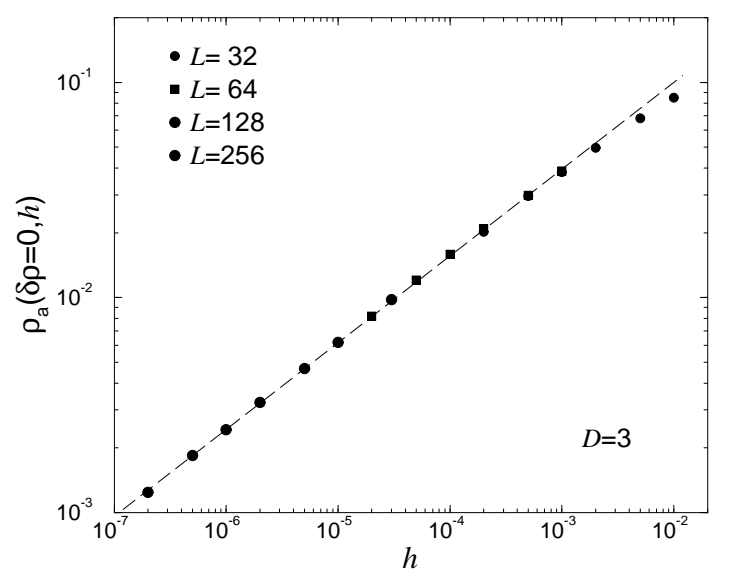

FIG. 5: The order parameter $\rho_{\mathrm{a}}$ as a function of the field $h$ at the critical density $\rho=\rho_{\mathrm{c}}$ for $D=3$. The dashed line corresponds to a power-law behavior according to Eq. (2) with an exponent $0.403 \pm 0.004$.

Additionally the fluctuation exponent $\gamma^{\prime}$ can be estimated via the scaling relation Eq. (6). Using the estimation $\nu_{\perp}=0.78 \pm 0.08$ we get $\gamma^{\prime}=0.286 \pm 0.161$ which again agrees with our result.

\section{B. $\mathrm{D}=3$}

Analogous to the two-dimensional case we first determine the exponent $\sigma$ for $D=3$. The field dependence

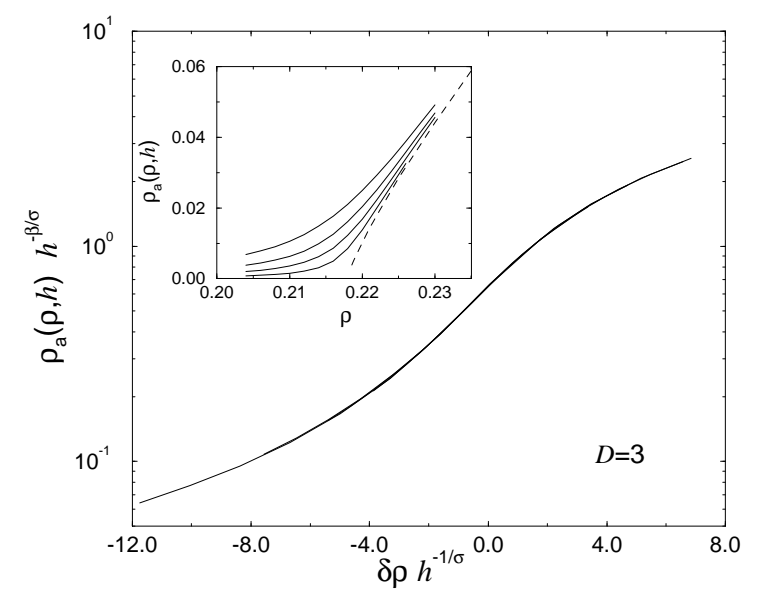

FIG. 6: The scaling plot of the order parameter $\rho_{\text {a }}$ for the three-dimensional model. The data are rescaled according to Eq. (11) with $\beta=0.837$ and $\sigma=2.075$. The inset displays the unscaled data, i.e., the order parameter $\rho_{\mathrm{a}}$ is plotted as a function of the density $\rho$ for different values of the field $h$. The dashed line corresponds to the zero-field behavior.

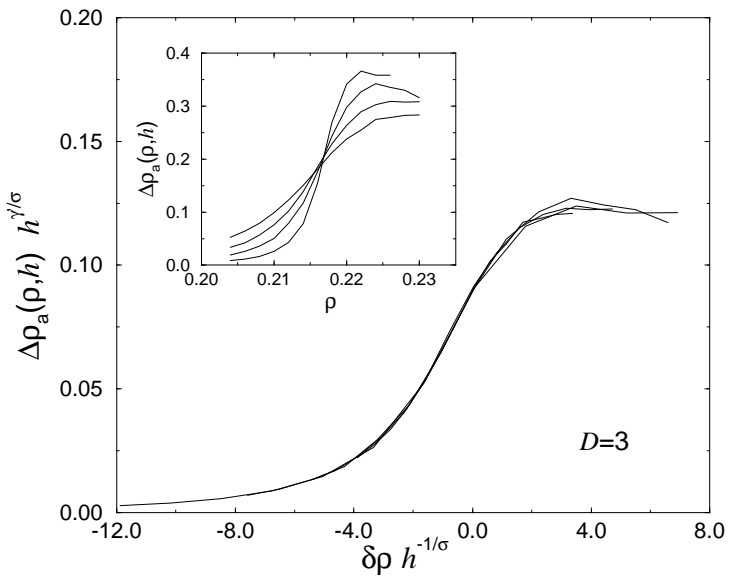

FIG. 7: The scaling plot of the order parameter fluctuations $\Delta \rho_{\mathrm{a}}$ for the three-dimensional model. The data are rescaled according to Eq. (12) with $\sigma=2.075$ and $\gamma^{\prime} / \sigma=0.1$. The inset displays the unscaled data, i.e., the fluctuations are plotted as a function of the density $\rho$ for different values of the field $h$.

of the order parameter is plotted in Fig. 5 . A regression analysis yields $\beta / \sigma=0.403 \pm 0.004$ which leads to the estimation $\sigma=2.075 \pm 0.043$ if one uses the value $\beta=0.837 \pm 0.015$ 纳.

In order to investigate the scaling behavior of the order parameter we simulated the CLG model for field values from $h=210^{-5}$ up to $h=210^{-4}$. The corresponding curves as well as the zero-field order parameter curve is shown in the inset of Fig. 6. According to Eq. (11) we plot the rescaled order parameter in the same figure. Using $\beta=0.837$ and $\sigma=2.075$ we get again and excellent data collapse of the different curves.

Furthermore we analyse the scaling behavior of the fluctuations $\Delta \rho_{\mathrm{a}}(\delta \rho, h)$ for the three-dimensional CLG model. The corresponding data are presented in the inset of Fig.77. Similar to the two-dimensional case one tries to obtain a data collapse of these curves by varying the exponent $\gamma^{\prime} / \sigma$ [Eq. (12)]. The best result is obtained for $\gamma^{\prime} / \sigma=0.10 \pm 0.02$ (see Fig. (7) which leads to $\gamma^{\prime}=0.208 \pm 0.042$.

Previous investigations of the fluctuations $\Delta \rho_{\mathrm{a}}$ for zero-field showed that $\Delta \rho_{\mathrm{a}}$ diverges for $\delta \rho \rightarrow 0$. But the numerical data could be interpreted either as a power-law divergence [Eq. (5)] with an exponent $\gamma^{\prime}=0.18 \pm 0.06$ or as a logarithmic growth [4. Our new results show that the fluctuations of the three-dimensional CLG model diverges algebraically at the transition point with the exponent $\gamma^{\prime}=0.208 \pm 0.042$. Additionally, the above results allows to estimate the correlation length exponents via the scaling relation Eq. (6) and one obtains $\nu_{\perp}=0.627 \pm 0.027$. 


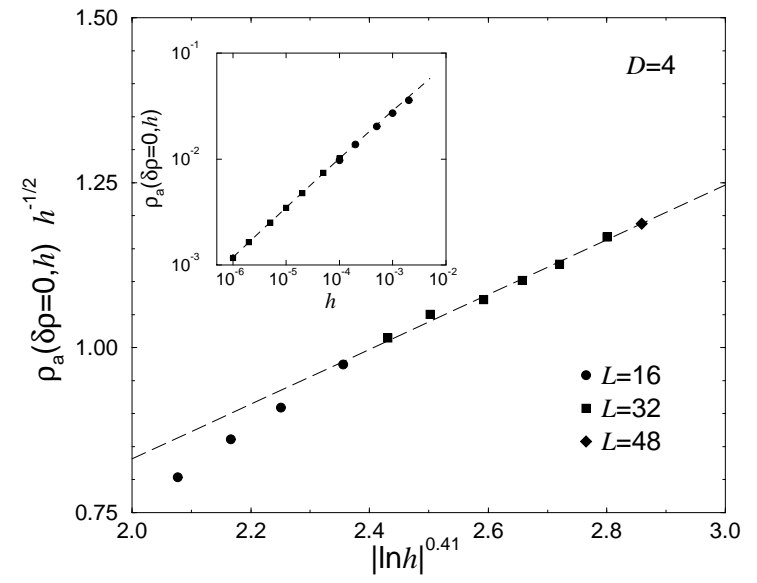

FIG. 8: The order parameter $\rho_{\mathrm{a}}$ as a function of the field $h$ at the critical density $\rho=\rho_{\mathrm{c}}$ for $D=4$. According to the scaling ansatz Eq. 15 we plot $\rho_{\mathrm{a}} h^{-1 / 2}$ as a function of $|\ln h|^{\Sigma}$. The expected asymptotic behavior $\rho_{\mathrm{a}} h^{-1 / 2}=$ const $|\ln h|^{\Sigma}$ is observed for $\Sigma=0.41$ (dashed line). The inset displays the original data. The dashed line corresponds again to the ansatz Eq. (15) with $\Sigma=0.41$.

\section{AT THE CRITICAL DIMENSION}

At the upper critical dimension $D=4$ the scaling behavior of the CLG model is affected by logarithmic corrections 沟, i.e., the scaling ansatz Eq. (3) has to be modified. Motivated by the scaling behavior of the Ising model we assume that the order parameter obeys in leading order the ansatz (see Appendix)

$$
\rho_{\mathrm{a}}(\delta \rho, h)=\lambda_{|\ln \lambda|^{l} \tilde{r}\left(\delta \rho \lambda^{-1 / \beta}|\ln \lambda|^{b}, h \lambda^{-\sigma / \beta}|\ln \lambda|^{s}\right),}
$$

where the exponents $\beta$ and $\sigma$ are given by the corresponding mean-field values $\beta=1$ and $\sigma=2$, respectively. Thus, for zero field the asymptotic scaling behavior of the order parameter obeys

$$
\rho_{\mathrm{a}}(\delta \rho, h=0) \sim \delta \rho|\ln \delta \rho|^{\mathrm{B}}
$$

with $\mathrm{B}=b+l$. This behavior was already observed for the CLG model with the logarithmic correction exponent $\mathrm{B}=0.24$ [8].

According to the above scaling ansatz the asymptotic field dependence of the order parameter at the critical density is given by

$$
\rho_{\mathrm{a}}(\delta \rho=0, h) \sim h^{1 / 2}|\ln h|^{\Sigma}
$$

with $\Sigma=s / 2+l$. In our analysis we plot $\rho_{\mathrm{a}} h^{-1 / 2}$ as a function of $|\ln h|^{\Sigma}$ and vary the exponent $\Sigma$ until one gets asymptotically a straight line. The best result is obtained for $\Sigma=0.41$ and the corresponding plot is shown in Fig. 8 .

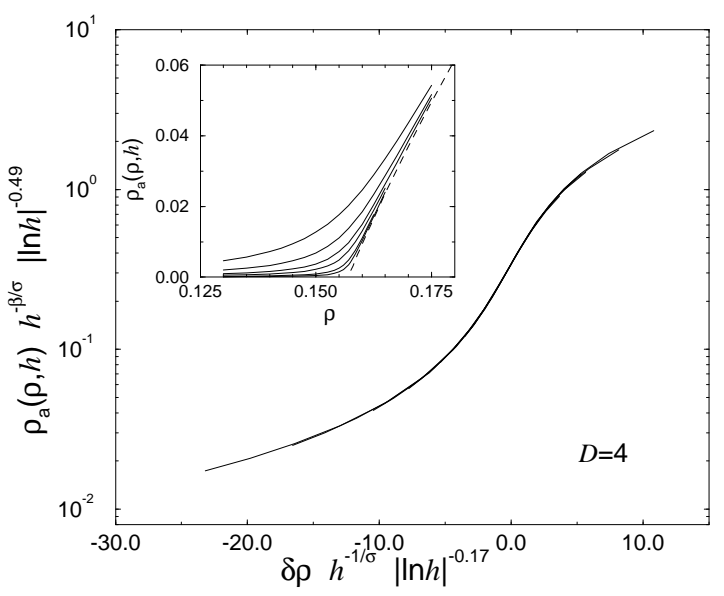

FIG. 9: The scaling plot of the order parameter $\rho_{\mathrm{a}}$ for the four-dimensional model, i.e., at the upper critical dimension. The data are rescaled according to Eqs. (16) 17) using the mean-field values $\beta=1$ and $\sigma=2$. The inset displays the unscaled data, i.e., the order parameter $\rho_{\mathrm{a}}$ is plotted as a function of the density $\rho$ for different values of the field $h$. The dashed line corresponds to the zero-field behavior.

Similar to the lower dimensions we consider the scaling behavior of the order parameter as a function of the control parameter for different external fields. In the inset of Fig. 9 we plot $\rho_{\mathrm{a}}$ vs. $\rho$ for different fields from $h=10^{-5}$ up to $h=510^{-4}$. Choosing $h \lambda^{-\sigma / \beta}|\ln \lambda|^{s}=1$ the scaling ansatz [Eq. (13)] yields in leading order

$$
\rho_{\mathrm{a}}(\delta \rho, h)=h^{1 / 2}|\ln h|^{\Sigma} \tilde{r}(x, 1),
$$

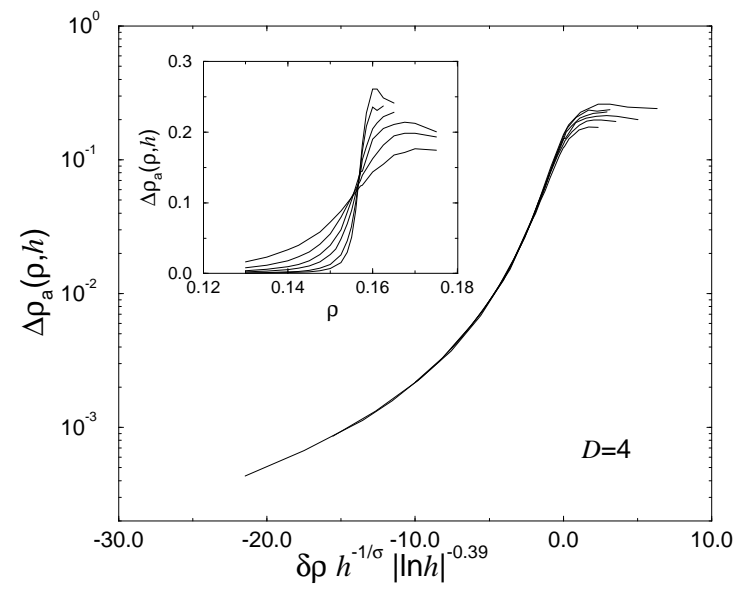

FIG. 10: The scaling plot of the order parameter fluctuations $\Delta \rho_{\mathrm{a}}$ for the four-dimensional model. The data are rescaled according to Eq. (19) using the mean-field value $\sigma=2$. The inset displays the unscaled data, i.e., the fluctuations are plotted as a function of the density $\rho$ for different values of the field $h$. 


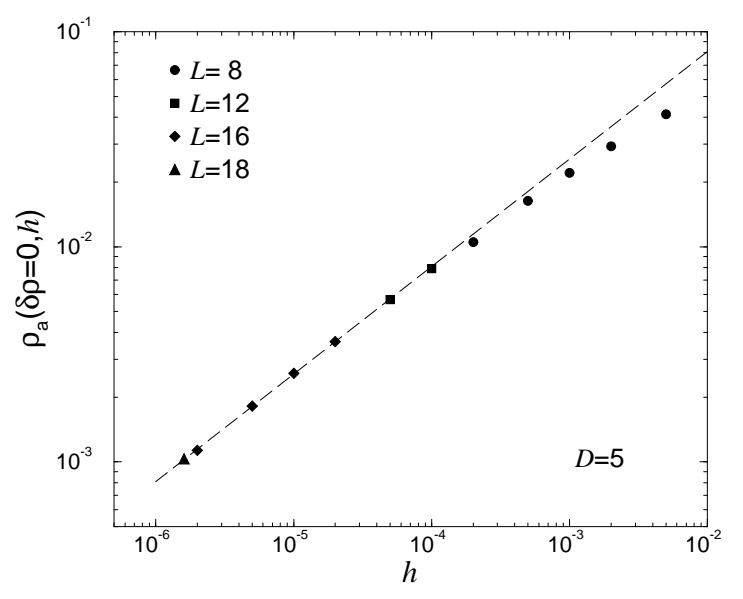

FIG. 11: The order parameter $\rho_{\mathrm{a}}$ as a function of the field $h$ at the critical density $\rho=\rho_{\mathrm{c}}$ for $D=5$. The dashed line corresponds to a power-law behavior according to Eq. (2) with $\beta / \sigma=1 / 2$.

where the scaling argument $x$ is given in leading order by

$$
x=\delta \rho h^{-1 / 2}|\ln h|^{b-s / 2}
$$

Varying the logarithmic correction exponents one gets a convincing data-collapse, which is shown in Fig. 9, for $\Sigma=0.49$ and $b-s / 2=-0.17$. The first value is in good agreement with $\Sigma=0.41$ obtained from the scaling behavior at the critical density [Eq. (15)]. Using the average value $\Sigma=l+s / 2=0.45$ and $b-s / 2=-0.17$ we get the estimation $B=b+l=0.28$ which agrees with $B=0.24$ obtained from numerical simulations in zero-field.

Furthermore we consider how the logarithmic corrections affect the scaling behavior of the fluctuations at the upper critical dimension. Similar to the order parameter [Eqs. (16,17)] we assume for the leading order of the fluctuations the scaling behavior

$$
\Delta \rho_{\mathrm{a}}(\delta \rho, h)=h^{-\gamma^{\prime} / \sigma}|\ln h|^{\Gamma} \tilde{d}\left(\delta \rho h^{-1 / \sigma}|\ln h|^{-\eta}, 1\right)
$$

with $\sigma=2$. It is known that the mean-field value of the fluctuation exponent is $\gamma^{\prime}=0$ which corresponds to a finite jump of $\Delta \rho_{\mathrm{a}}$ at the critical density [4, 5]. In order to avoid that $\Delta \rho_{\text {a }}$ diverges for $h \rightarrow 0$ at $\delta \rho=0$ the logarithmic correction exponent $\Gamma$ has to be set to zero too. Therefore we try to obtain a data collapse of the fluctuation data according to the ansatz

$$
\Delta \rho_{\mathrm{a}}(\delta \rho, h)=\tilde{d}\left(\delta \rho h^{-1 / 2}|\ln h|^{-\eta}, 1\right) .
$$

A good data collapse is observed for $\eta=0.39$ and the corresponding plot is shown in Fig. 10 .

\section{ABOVE THE CRITICAL DIMENSION}

Above the critical dimension, i.e. $D \geq 5$, the scaling behavior of the CLG model is expected to obey again the

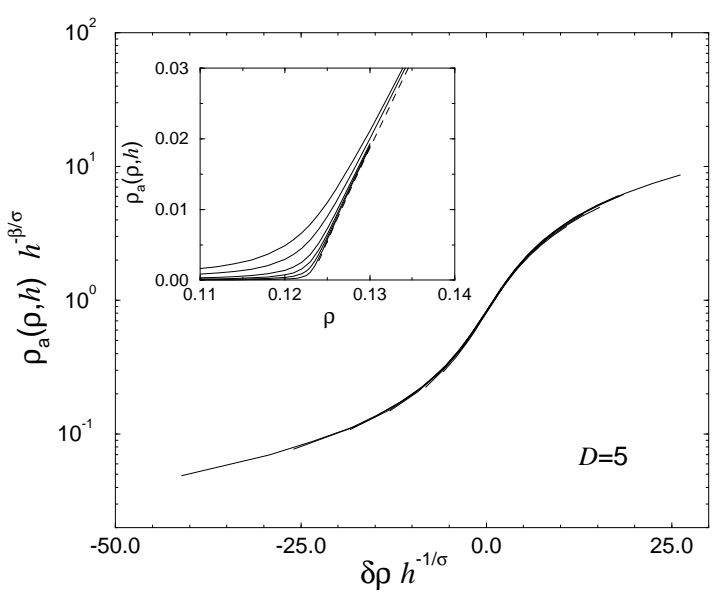

FIG. 12: The scaling plot of the order parameter $\rho_{\mathrm{a}}$ for the five-dimensional model, i.e., above the upper critical dimension. The data are rescaled according to Eq. (11) using the mean-field values $\beta=1$ as well as $\sigma=2$. The inset displays the unscaled data, i.e., the order parameter $\rho_{\mathrm{a}}$ is plotted as a function of the density $\rho$ for different values of the field $h$. The dashed line corresponds to the zero-field behavior.

scaling ansatz Eq. (3) where the exponents are given by the mean-field values $\beta=1, \sigma=2$, and $\gamma^{\prime}=0$ [4, 5, independently of the particular dimension.

In Fig.11 we plot the field dependence of the order parameter at the critical density. For sufficiently small values of the field $\left(h \leq 10^{-4}\right)$ we observe the expected scaling behavior $\rho_{\mathrm{a}} \sim h^{1 / 2}$. Thus the order parameter

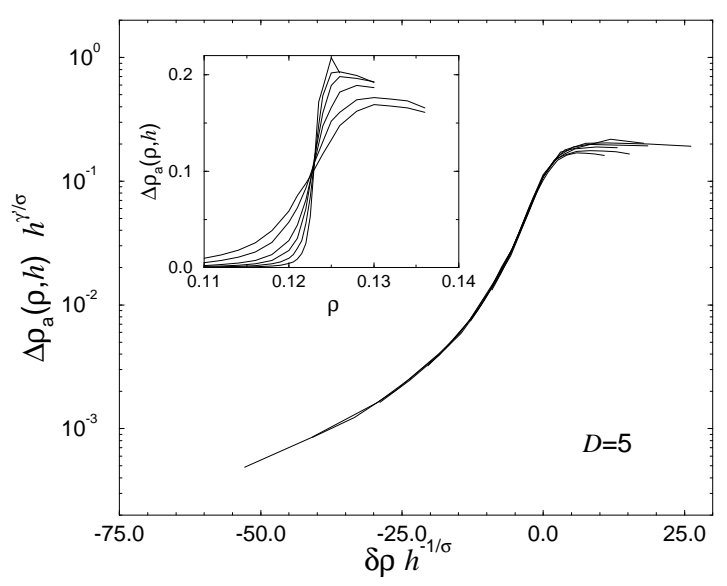

FIG. 13: The scaling plot of the order parameter fluctuations $\Delta \rho_{\mathrm{a}}$ for the five-dimensional model. The data are rescaled according to Eq. (12) using the mean-field values $\sigma=2$ and $\gamma^{\prime}=0$. The inset displays the unscaled data, i.e., the fluctuations are plotted as a function of the density $\rho$ for different values of the field $h$. 
TABLE I: The critical density $\rho_{\mathrm{c}}$ and the critical exponents $\beta, \sigma \gamma^{\prime}$ and $\gamma$ of the CLG model for various dimensions $D$. The values of the susceptibility exponent $\gamma$ are calculated via Eq. (10). The symbol * denotes logarithmic corrections to the power-law behavior. The values of $\rho_{\mathrm{c}}$ and $\beta$ are obtained from [ [-

\begin{tabular}{llllll}
$D$ & $\rho_{\mathrm{c}}$ & $\beta$ & $\sigma$ & $\gamma^{\prime}$ & $\gamma$ \\
\hline & & & & \\
2 & 0.34494 & $0.637 \pm 0.009$ & $2.227 \pm 0.032$ & $0.384 \pm 0.023$ & $1.590 \pm 0.033$ \\
3 & 0.21791 & $0.837 \pm 0.015$ & $2.075 \pm 0.043$ & $0.208 \pm 0.042$ & $1.238 \pm 0.046$ \\
4 & 0.15705 & $1^{*}$ & $2^{*}$ & $0^{*}$ & $1^{*}$ \\
5 & 0.12298 & 1 & 2 & 0 & 1
\end{tabular}

$\rho_{\mathrm{a}}(\delta \rho, h)$ is determined for various fields in this regime (from $h=210^{-6}$ up to $10^{-4}$ ). The curves are shown in the inset of Fig. 12. Rescaling these curves according to the ansatz Eq. (3) with $\beta=1$ and $\sigma=2$ one gets a good data collapse (see Fig. 12).

Finally we consider the fluctuations of the fivedimensional CLG model. The inset of Fig. 13 shows how the fluctuations are affected by the field $h$. With vanishing field the curves become steeper until one gets a jump [4, 5] for $h=0$.

Considering the scaling behavior one has to taken into account that the jump corresponds to $\gamma^{\prime}=0$. Therefore, we plot the fluctuations $\Delta \rho_{\mathrm{a}}$ as a function of $\delta \rho h^{-1 / 2}$. The resulting data collapse is shown in Fig. 13 and confirms the assumed scaling behavior.

\section{CONCLUSIONS}

We introduced a method which allows to apply an external field in the CLG model. The external field obeys the particle conservation and is conjugated to the order parameter, i.e., it is realized as a spontaneous creation of active particles. We considered the order parameter as well as its fluctuations of the CLG model as a function of an external field in various dimensions $(D=2,3,4,5)$. Although the external field drives the system away from criticality the order parameter obeys certain scaling laws for sufficiently small values of the external field. These scaling laws are investigated and the corresponding exponents are determined numerically. The obtained values of the field exponent $\sigma$ are listed together with other critical indices in Table I. At the upper critical dimension $D_{\mathrm{c}}=4$ the usual scaling behavior has to be modified by additional logarithmic corrections.

\section{Acknowledgments}

I would like to thank A. Hucht for helpful discussions and useful comments on the manuscript.

\section{APPENDIX}

Recently, the scaling behavior of the well known Ising model was investigated at the upper critical dimension $\left(d_{\mathrm{c}}=4\right)$ and it was argued that the singular part of the free energy obeys the finite-size scaling ansatz [9]

$$
f_{L}(t, h)=L^{-4} \tilde{f}\left(t L^{2} \ln ^{1 / 6} L, h L^{3} \ln ^{1 / 4} L\right)
$$

where $t$ denotes the reduced temperature $\left(t=T / T_{\mathrm{c}}-1\right)$, $h$ an applied magnetic field and $L$ denotes the system size. Thus we assume that the free energy is a generalized homogeneous function

$$
f(t, h, L)=\begin{aligned}
& (21) \\
& \lambda \tilde{f}\left(t \lambda^{-1 / 2}|\ln \lambda|^{1 / 6}, h \lambda^{-3 / 4}|\ln \lambda|^{1 / 4}, L \lambda^{1 / 4}\right)
\end{aligned}
$$

with $\lambda>0$. Of course for $\lambda=L^{-4}$ one recovers Eq. (2q). This ansatz can be checked in the following way: the derivative of the free energy with respect to the applied field leads to the scaling equation of the magnetization

$$
\begin{array}{r}
m(t, h, L)=\lambda^{1 / 4}|\ln \lambda|^{1 / 4} \\
\tilde{m}\left(t \lambda^{-1 / 2}|\ln \lambda|^{1 / 6}, h \lambda^{-3 / 4}|\ln \lambda|^{1 / 4}, L \lambda^{1 / 4}\right) .
\end{array}
$$

Choosing $t \lambda^{-1 / 2}|\ln \lambda|^{1 / 6}=1$ one gets in leading order for the order parameter in the thermodynamic limit at zero field

$$
m(t, h=0) \sim t^{1 / 2}|\ln t|^{1 / 3} .
$$

The field dependence of the magnetization at the critical temperature $(t=0)$

$$
m(t=0, h) \sim h^{1 / 3}|\ln h|^{1 / 3}
$$

is obtained by setting $h \lambda^{-3 / 4}|\ln \lambda|^{1 / 4}=1$. Analogous one gets in leading order for the susceptibility and specific heat

$$
\chi(t, h=0) \sim t^{-1}|\ln t|^{1 / 3}
$$

and

$$
c(t, h=0) \sim|\ln t|^{1 / 3},
$$

respectively. In this way the ansatz Eq. (21) leads directly to the Eqs. 23, 24, 25,26) which were already derived in 
the 1970s by Wegner and Riedel using renormalization group techniques [10. It is worth to mention that these are exact results within the renormalization group theory, i.e., neither the values of the mean-field exponents nor the values of logarithmic correction exponents are obtained from approximation schemes like $\epsilon$ - or $1 / n$-expansions.

In the case of the CLG model we choose a scaling ansatz for the leading order of the order parameter [Eq. (13)] with corresponds to Eq. (22).
[1] M. Rossi, R. Pastor-Satorras, and A. Vespignani, Phys. Rev. Lett. 85, 1803 (2000).

[2] H. K. Janssen, Z. Phys. B 42, 151 (1981).

[3] P. Grassberger, Z. Phys. B 47, 365 (1982).

[4] S. Lübeck, Phys. Rev. E 64, 016123 (2001).

[5] S. Lübeck and A. Hucht, J. Phys. A 34, L577 (2001).

[6] H. Hinrichsen, Adv. Phys. 49, 815 (2000).

[7] I. Jensen and R. Dickman, Phys. Rev. E 48, 1710 (1993).
[8] The logarithmic correction exponent $\mathrm{B}=0.39$ was determined in [Th using the ansatz $\rho_{\mathrm{a}} \sim\left(\rho-\rho_{\mathrm{c}}\right)\left|\ln \left(\rho-\rho_{\mathrm{c}}\right)\right|^{\mathrm{B}}$. Fitting the same data to the different ansatz Eq. (14) one gets the value $\mathrm{B}=0.24$.

[9] N. Aktekin, J. Stat. Phys. 104, 1397 (2001).

[10] F. J. Wegner and E. K. Riedel, Phys. Rev. B 7, 248 (1973). 